\title{
Integration of mental health into priority health service delivery platforms: maternal and child health services
}

\author{
A. Rahman ${ }^{7}$
}

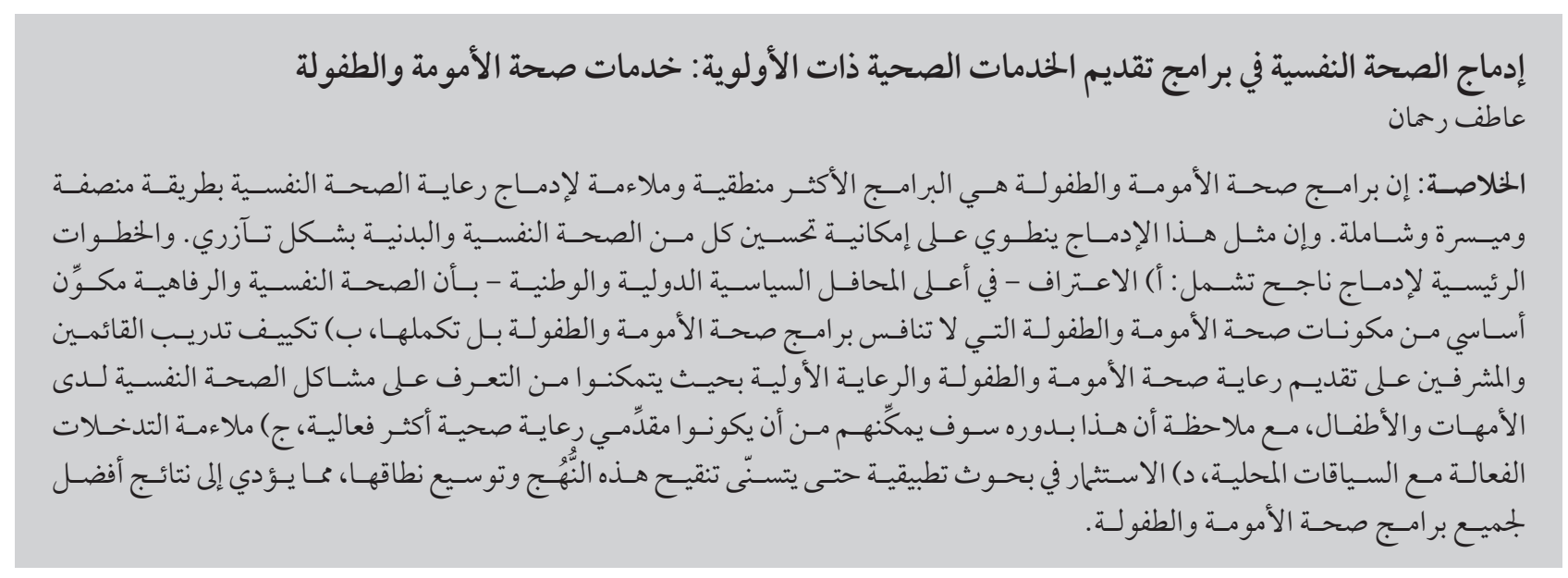

ABSTRACT Maternal and child health $(\mathrm{MCH})$ programmes are the most logical and appropriate platforms for integration of mental health care in an equitable, accessible and holistic manner. Such integration has the potential to improve both mental and physical health synergistically. Key steps to successful integration include a) recognition, at the highest international and national policy forums, that mental health and well-being is a generic component of $\mathrm{MCH}$ that does not compete with $\mathrm{MCH}$ programmes but instead complements them; b) tailoring the training and supervision of $\mathrm{MCH}$ and primary care personnel so they can recognize and assist in the management of common maternal and child mental health problems, recognizing that this, in turn, will enable them to be more effective health-care workers; c) adapting effective interventions to local contexts; and d) investing in implementation research so that these approaches are refined and scaled-up, leading to improved outcomes for all MCH programmes.

Intégration de la santé mentale dans des plateformes de prestation de services de santé prioritaires : services de santé de la mère et de l'enfant

RÉSUMÉ Les programmes de santé de la mère et de l'enfant sont les plateformes les plus logiques et adéquates pour l'intégration de soins de santé mentale d'une manière équitable, accessible et holistique. Une telle intégration a le potentiel d'améliorer la santé mentale et physique de manière synergique. Les étapes clés pour une intégration réussie sont les suivantes : a) reconnaître, dans les forums politiques nationaux et internationaux de très haut niveau que la santé mentale et le bien-être sont des composantes génériques de la santé de la mère et de l'enfant, qui ne font pas concurrence aux programmes de santé de la mère et de l'enfant mais viennent plutôt les complèter ; b) adapter la formation et la supervision du personnel de soins de santé primaires et de santé de la mère et de l'enfant pour qu'il identifie les problèmes courants dans ce domaine et contribue à leur prise en charge, reconnaissant que cette démarche leur permettra de devenir des agents de santé plus efficaces ; c) adapter des interventions efficaces aux contextes locaux; et d) investir dans la recherche sur la mise en ouvre pour que ces méthodes soient affinées et intensifiées, afin d'améliorer les résultats de tous les programmes de santé de la mère et de l'enfant. 


\section{Why integration?}

Mental health problems affect $10-20 \%$ of children and adolescents and $15-20 \%$ of mothers worldwide, and account for a large portion of the global burden of disease. The most logical and appropriate platforms for integration of interventions to prevent or manage many such problems are community-based maternal and child health $(\mathrm{MCH})$ services $(1)$. There are a number of advantages to such integration $(1,2)$, as outlined below.

1. Effective treatments exist for most common maternal and child mental health problems $(3,4)$, but few patients have access to such treatments. In many low- and middle-income countries, the ratio of specialists to population is 1:0.5 million and in some, as low as 1:4 million. Even in developed countries only 2 in 10 adults, and an even lower number of children, with common mental health problems receive care from a mental health specialist in any given year. As $\mathrm{MCH}$ programmes are population and community-based, these are more likely to provide equitable care, especially to rural and difficultto-access communities.

2. Integrated treatment programmes in which health and social care providers are supported to manage common mental health problems offer a chance to treat the whole patient, an approach that is more patient-centred and is often more effective than one in which mental, physical and reproductive health problems are each addressed in different so-called "silos", without effective communication between providers $(1,2)$. Community health workers (CHWs), especially $\mathrm{MCH}$ workers, are best placed to adopt an ecological approach to care. This is particularly important for children, whose psychosocial wellbeing is closely linked to the mental health of the parents and the quality of the family and school environment.
These workers have a knowledge of community resources and health and social and education services, and can better respond to the specific needs of local communities.

3. Integrated care programmes that can address maternal and child mental health needs in the context of $\mathrm{MCH}$ care settings are often more attractive to patients and family members who are concerned about the stigma associated with mental and developmental disorders and about mental health treatment settings.

4. Integration with $\mathrm{MCH}$ platforms is suitable for taking into account mental health needs across the life-course. Frontline $\mathrm{MCH}$ workers can establish trusting and long-term relationships with children and families and prevent mental health problems by promoting healthy lifestyles and by providing early identification and timely preventive and curative interventions for common behavioural, emotional and social problems in children and for risk factors in the perinatal period.

5. There are benefits too for $\mathrm{MCH}$ programmes themselves when there is integration with mental health care services. For example, treating maternal depression can improve the capacity of mothers to be more receptive to $\mathrm{MCH}$ programmes, improve their ability to care for their children, and improve mother-infant interaction.

\section{Key steps for integrating maternal and child mental health into $\mathrm{MCH}$ platforms}

The following steps could facilitate integration of maternal and child mental health into $\mathrm{MCH}$ platforms $(1,2)$.

\section{Engaging stakeholders}

Stakeholders need to agree that mental health interventions within maternal and child health platforms advances maternal and child health, and frontline clinicians must see the value of adding these interventions to their current services. Joint assessment by the managers of the priority health programmes and by mental health professionals and service planners also enhances ownership and commitment to achieve the planned outcomes within agreed timelines.

\section{Analysing goals, functions and resources}

The next step is a detailed analysis of the goals, functions and resources (human and financial) of the CHW or $\mathrm{MCH}$ programme in which mental health care will be integrated. This step should include attention to the existing knowledge and skills of health-care providers about identification and care of common mental health problems; recognition of when to refer; inclination/motivation to enhance their skills; and the perceived benefits of these skills to advance their professional and programmatic goals.

\section{Identifying shared and achievable objectives}

Identifying shared and achievable objectives requires joint assessment of the needs and feasibility of integration; the identification of key tasks; and the training, support, and supervision needed for clinicians to provide these interventions. Attention must be paid to congruence of the integration efforts with the overall objectives of the priority programmes and to the resources needed to ensure initial success and sustainability. It is advisable to begin with limited but clear and specific objectives. For example, the initial target for integration of maternal mental health care within $\mathrm{MCH}$ programmes may be the identification and management of maternal depression to achieve better infant nutritional and development outcomes (5) (see Box 1). 


\section{Changing the role of mental health specialists}

A change in the role of specialist mental health professionals from providers of individual care to consultants is needed for adaptation and implementation of mental health interventions and for training and supervision of $\mathrm{CHW}$ and $\mathrm{MCH}$ workers.

\section{Developing effective intervention programmes}

Effective intervention programmes need to be developed with skills-sets that logically group together in terms of content, training and operational use. Box 1 illustrates a case study from Pakistan that attempts this with an intervention for maternal psychosocial well-being integrated into a child nutrition and early development programme. Most of the required functions can be performed by a range of workers, many of who are already part of $\mathrm{MCH}$ services, thus allowing some flexibility in planning and adaptation and marginal additional investments. Particular skills, such as behaviour-change communication, motivational coaching, patient education and self-management support can be critical for providing effective $\mathrm{MCH}$ care. These care management tasks or work packages can be effectively assigned to non-specialist health workers who are well-positioned to bring them into the community, thus extending the reach of primary care. Such intervention programmes should be tailored to a lifecourse approach, integrating with the stages of preconception, perinatal and early childhood through to adolescence and early adulthood.

\section{Assigning responsibilities and establishing a monitoring mechanism}

Clear and explicit responsibilities need to be assigned to the health-care providers and managers of the priority programmes and to the mental health team at each level. Flowcharts and referral algorithms, such as the World Health Organization's Mental Health Gap Action Programme (mhGAP)

\section{Box 1 Case study: Five-Pillars (5-PA) Approach to Maternal Psychosocial Well-Being}

5-PA is derived from the Thinking Healthy Programme (THP), a cognitive-behaviour therapy-based psychosocial intervention for mothers with depression and their infants. THP was a targeted intervention for women suffering from perinatal depression and their infants and was delivered by community health workers (CHW) in rural Rawalpindi, Pakistan. In a randomized controlled trial to evaluate the approach there were impressive improvements in maternal depression and functioning compared with controls (6), and THP was adopted by the World Health Organization as a first-line low-intensity treatment for perinatal depression (see http://www.who.int/mental_health/maternal-child/thinking_healthy/en/)

5-PA is an adaptation of the THP to integrate it into a child nutrition and development programme (5). Thus, it is an example of the delivery of mental health care to mothers and infants through an $\mathrm{MCH}$ platform. The adaptation targets not only depressed women but all mothers during pregnancy and in the 2 years after giving birth.

The key feature of the approach is that it is integrated into, and facilitates the delivery of, a CHW-delivered intervention for early child nutrition and development. Thus, whenever the CHW delivers a session for child nutrition or development, she uses the 5P approach to both strengthen the key message as well as provide the psychosocial intervention. In practice, the approach works as follows:

Pillar 1. Family support. An initial home visit emphasizes family participation, and the training manual gives specific instructions on how this can be facilitated. Family members are encouraged to be active partners for the whole duration of the programme. Strategies to engage key decision-makers, such as mothers-in-law and husbands, are emphasized.

Pillar 2. Empathic listening. Each session begins in an open-ended fashion, with the CHW allowing the woman to talk freely. She uses active listening skills to convey empathy and makes a list of problems the woman faced in performing the desired behaviours that the CHW might have suggested in her previous visit.

Pillar 3. Guided discovery using pictures. Each new health message related to play, stimulation or nutrition is conveyed using this approach. Using carefully researched pictures, the CHW discusses both undesired and desired behaviours. She is trained not to impose her views but to allow the mother and family to consider each viewpoint and come to their own conclusions. The idea is that the basis of any behaviour change begins at the cognitive level.

Pillar 4. Behavioural activation. Once the message is received and accepted, the activities related to it have to be made manageable so that a sense of mastery is achieved. The training manual has suggestions for how each nutrition or play-related task can be broken down and monitored with the help of family members.

Pillar 5. Problem-solving. The CHW spends time discussing the problems the woman faced in carrying out the tasks suggested in the previous session (see Pillar 2). She discusses possible solutions, which she can generate through discussion with the family or through her supervision. 
intervention guide (7), can be very helpful in this step of planning. They can also then be linked to the monitoring mechanism using a limited number of clear, relevant and agreed-upon goals.

\section{Stepping-up intervention}

Intervention needs to be systematically adjusted - "stepped up" - if patients are not improving as expected with input from a specialist consultant. Patients who continue to show no response to treatment or have an acute crisis should be referred to mental health specialty care.

\section{Developing human resources}

Sustainable models need to be developed to train and increase the number of culturally and ethnically diverse lay and specialist providers to deliver evidence-based services. Capacity in lowand middle-income countries can be increased by creating regional centres for mental health research, education, training and practice that incorporate the views and needs of local people as well as a life-course approach.

\section{Addressing stigma related to mental illness}

Stigma related to mental illness that could impede the integration of mental health into $\mathrm{MCH}$ programmes can be addressed through community awareness programmes.

\section{Creating parity between mental and physical illness}

Parity can be created between mental and physical illness in terms of investment into research, training, treatment and prevention. This would be facilitated by incorporating a mental health component into international $\mathrm{MCH}$ aid and development programmes.

\section{Barriers and challenges}

Health-care systems vary in their ability to respond to national health care needs. Many health-care systems, and particularly those in fragile post-conflict settings, lack the core health system elements needed to provide the most basic set of services. Problems include poor financing and a fiscal infrastructure largely dependent on external aid; fragmentation of structures and services; weak systems for procurement (including inadequate supply of medications and poor or no access to diagnostic services); inadequate or weak governance and leadership; and a workforce that is often overwhelmed and experiencing high turnover. Integration may be the only feasible option to address mental health problems in the context of a weak health system, and doing so can contribute to systems strengthening more generally.

Policy-makers fear that mental health interventions will divert the energies of health-care staff and dilute the impact of other priority interventions. This view fails to take into account the holistic nature of health, and erroneously propagates the defunct theory of mind-body dualism. Most current evidence demonstrates the interconnectedness of physical and mental health and suggests that integrated interventions can achieve synergistic results.

A substantial obstacle to the integration of mental health care is lack of consensus over how to standardize and assign mental health-care tasks so that these can be delivered seamlessly by the same worker in a single programme. Consensus treatment packages, such as those in the mhGAP intervention guide, describe what counts as good and evidence-based care (7). But these packages need to be adapted for integration. For any health workforce to be effective, and for care packages to be delivered as intended, treatment guidelines need to be operationalized into coordinated roles and tasks. The starting point for effective integrated care pathways is to specify the skill-sets necessary to deliver integrated care effectively and to plan for the development and deployment of these skills in the context of available human resources.

A major barrier remains the social exclusion and negative attitudes attached to mental health problems. Overcoming this barrier is as much a challenge for the public health professionals as for the communities they serve.

If the goal of improving women's well-being from childhood through to old age is to be achieved, healthy policies aimed at improving the social status of women are needed along with health policies targeting the entire spectrum of women's health needs. Integration of maternal mental health into the $\mathrm{MCH}$ agenda can provide a universally acceptable window of opportunity for creating healthy policies, from education to economic empowerment to legal and political mechanisms that enhance the status of women.

For the post-2015 agenda, mental health and noncommunicable diseases are seen as a central focus by public health experts globally, with a paradigm shift towards a comprehensive discourse. An integrated approach adopting so-called "systems thinking" that is guided by evidence-based resource allocation would ensure a responsive health system that is able to meet the needs of women and children. However, there are still those who lobby for attention to areas such as survival, nutrition, communicable diseases and who continue to be fixated on a singular condition or risk (e.g. poverty) without considering the synergies of action that an integrated approach would bring.

There is inadequate recognition of the link between mental health and other health conditions. Insufficient connections are made between mental health problems and other health conditions and how these impact each other in mothers and children. Without making this connection there is a risk that the burden of mental health problems is underestimated and mental 
health care becomes alienated from mainstream efforts to improve health and reduce poverty.

\section{Conclusions}

This paper highlights the case for integrating maternal and child mental health interventions into mainstream $\mathrm{MCH}$ services. Such integration has the potential to improve both mental and physical health synergistically, with likely benefits throughout the life-course.
It discusses the key steps required and potential barriers to such integration. The responsibility to meet these challenges should be shared equally by mental health teams as well as the $\mathrm{MCH}$ community, and the following concrete measures should be taken as a matter of priority (1):

- Recognize, at the highest international and national policy forums, that mental health and well-being is a generic component of $\mathrm{MCH}$ that does not compete with $\mathrm{MCH}$ programmes but instead complements them.
- Tailor the training and supervision of $\mathrm{MCH}$ and primary care personnel so they can recognize and assist in the management of common maternal and child mental health problems. This, in turn, will enable them to be more effective health workers.

- Adapt effective interventions to local contexts and strengthen systems of supervision.

- Invest in research so that these approaches are refined and scaled-up, leading to improved outcomes to all $\mathrm{MCH}$ programmes.

\section{References}

1. Rahman A, Surkan PJ, Cayetano CE, Rwagatare P, Dickson KE, Dickson KE. Grand challenges: integrating maternal mental health into maternal and child health programmes. PLoS Med. 2013;10(5):e1001442. PMID:23667345

2. PatelV, Belkin GS, Chockalingam A, CooperJ, SaxenaS, Unützer J. Grand challenges: integrating mental health services into priority health care platforms. PLoS Med. 2013;10(5):e1001448. PMID:23737736

3. Rahman A, Fisher J, Bower P, Luchters S, Tran T, Yasamy MT, et al. Interventions for common perinatal mental disorders in women in low- and middle-income countries: a systematic review and meta-analysis. Bull World Health Organ. 2013 Aug 1;91(8):593-601. PMID:23940407

4. Kieling C, Baker-Henningham H, Belfer M, Conti G, Ertem $\mathrm{M}$, Omigbodun $\mathrm{O}$, et al. Child and adolescence mental health: evidence for action. Lancet. 2011;378(9801):1515-25. PMID:22008427
5. Zafar S, Sikander S, Haq Z, Hill Z, Lingam R, Skordis-Worrall $\mathrm{J}$, et al. Integrating maternal psychosocial well-being into a child-development intervention: the five-pillars approach. Ann N Y Acad Sci. 2014 Jan;1308:107-17. 10.1111/nyas.12339 PMID:24571213

6. Rahman A, Malik A, Sikander S, Roberts C, Creed F. Cognitive behaviour therapy-based intervention by community health workers for mothers with depression and their infants in rural Pakistan: a cluster-randomised controlled trial. Lancet. 2008 Sep 13;372(9642):902-9. PMID:18790313

7. Dua T, Barbui C, Clark N, Fleischmann A, Poznyak V, van Ommeren M, et al. Evidence-based guidelines for mental, neurological, and substance use disorders in low- and middleincome countries: summary of WHO recommendations. PLoS Med. 2011 Nov;8(11):e1001122. PMID:22110406 